\title{
Electrophoretic Mobility of a Sphere in a Spherical Cavity
}

\author{
Eric Lee, Jhih-Wei Chu, and Jyh-Ping Hsu ${ }^{1}$ \\ Department of Chemical Engineering, National Taiwan University, Taipei, Taiwan 10617, Republic of China
}

Received January 7, 1998; accepted April 17, 1998

\begin{abstract}
The electrophoretic behavior of a spherical particle in a spherical cavity is analyzed theoretically, taking the effect of double layer polarization into account. We show that for the case where the particle is positively charged and the cavity uncharged if the surface potential of particle is high, the variation of the mobility of the particle as a function of $\kappa a$ has a minimum, $\kappa$ and $a$ being respectively the reciprocal $D$ ebye length and particle radius. This minimum does not appear if the effect of double layer polarization is neglected. The variation of the mobility as a function of $\kappa a$ has a minimum for a medium value of $\lambda(=$ particle radius/cavity radius); it becomes negligible if $\lambda$ is either small or large. In the case where the particle is uncharged and the cavity positively charged, if the surface potential is high, the variation of mobility as a function of $\kappa a$ has a maximum; if it is low, the mobility increases monotonically with $\kappa a$. Here, the mobility is mainly determined by the drag force, rather than by the electric force, acting on the particle as in the case where the particle is positively charged and the cavity uncharged. If both the particle and the cavity are charged, the electrophoretic behavior of the particle can be deduced from the results of the above two cases. $\odot 1998$ Academic Press
\end{abstract}

Key Words: electrophoresis; mobility; double layer polarization; spherical charged particle; boundary effect; spherical charged cavity.

\section{INTRODUCTION}

Knowledge about the surface potential of a charged entity is essential to various applications in practice. Typical example includes the adsorption of colloidal particles to a rigid surface and the coagulation behavior of a colloidal suspension (1). In practice, the surface potential is usually characterized by the zeta potential, the electrical potential at the shear plane of the electrical double layer near the charged entity which can be estimated on the basis of the electrophoretic mobility of the entity in an applied electrical field (1). The determination of the relation between the electrophoretic mobility and the zeta potential involves the solution of a set of differential equations, known as the electrokinetic equations (2). Due to its complicated nature, solving this set of equations analytically is almost impossible, in general, except for some limiting cases, in which

\footnotetext{
${ }^{1}$ To whom correspondence should be addressed. E-mail: t8504009 @ ccms.ntu.edu.tw.
}

the electrokinetic equations can be simplified drastically. For an isolated sphere in an infinite fluid, for example, an analytical expression for the electrophoretic mobility as a function of zeta potential can be retrieved if the electrical potential is low, and the double layer is either very thin (3-6) or very thick (7) relative to particle radius. The effect of double layer distortion due to the presence of the applied electric field on the electrophoretic mobility was neglected in these studies. A numerical procedure is necessary if this effect is taken into account. The numerical solution for the electrokinetic equations was first provided by Wiersema et al. (8). O'Brien and White (9) suggested an effective numerical scheme for the resolution of the linearized electrokinetic equations. Their approach is valid for the case where the applied field is weak relative to that induced in the double layer around a particle. The electrophoretic behavior of a polyion in an infinite fluid was examined recently by Allison and Nambi (10); the governing equations were solved by a combined DIE/finite difference algorithm. The problem was also solved by using a boundary element method (11). They concluded that the accuracy of the spatial variation of electrical potential and ion density is crucial in the estimation of the electrophoretic mobility of a charged entity.

In practical applications, electrophoresis is almost always conducted in a finite domain, which implies that the boundary effect on the electrophoretic behavior of a particle may be significant. This effect makes the analysis much more complicated than that if the boundary is absent. A considerable amount of effort was made recently to examine the electrophoresis in a bounded region (e.g., 12-15). Keh and Anderson (13) used the method of reflections to analyze the electrophoretic motion of a particle normal to a large conductive plane, parallel to a large dielectric plane, along the axis of a long cylinder, and along the centerline between two large parallel plates. It was concluded that the results of Keh and Anderson are satisfactory for $\lambda<0.7$ (15-17), $\lambda$ being the ratio (linear size of particle/linear size of boundary). The electrophoresis of a sphere through the center of a circular disk (18), that of an infinite insulating cylinder parallel to a planar wall (19), and that of an arbitrary prolate body of revolution towards an infinite conducting wall (20) were studied theoretically. All of these are based on the assumptions that the electrical double layer around a charged particle is infinitely thin relative to its linear size, and the electrical potential is low. In general, 


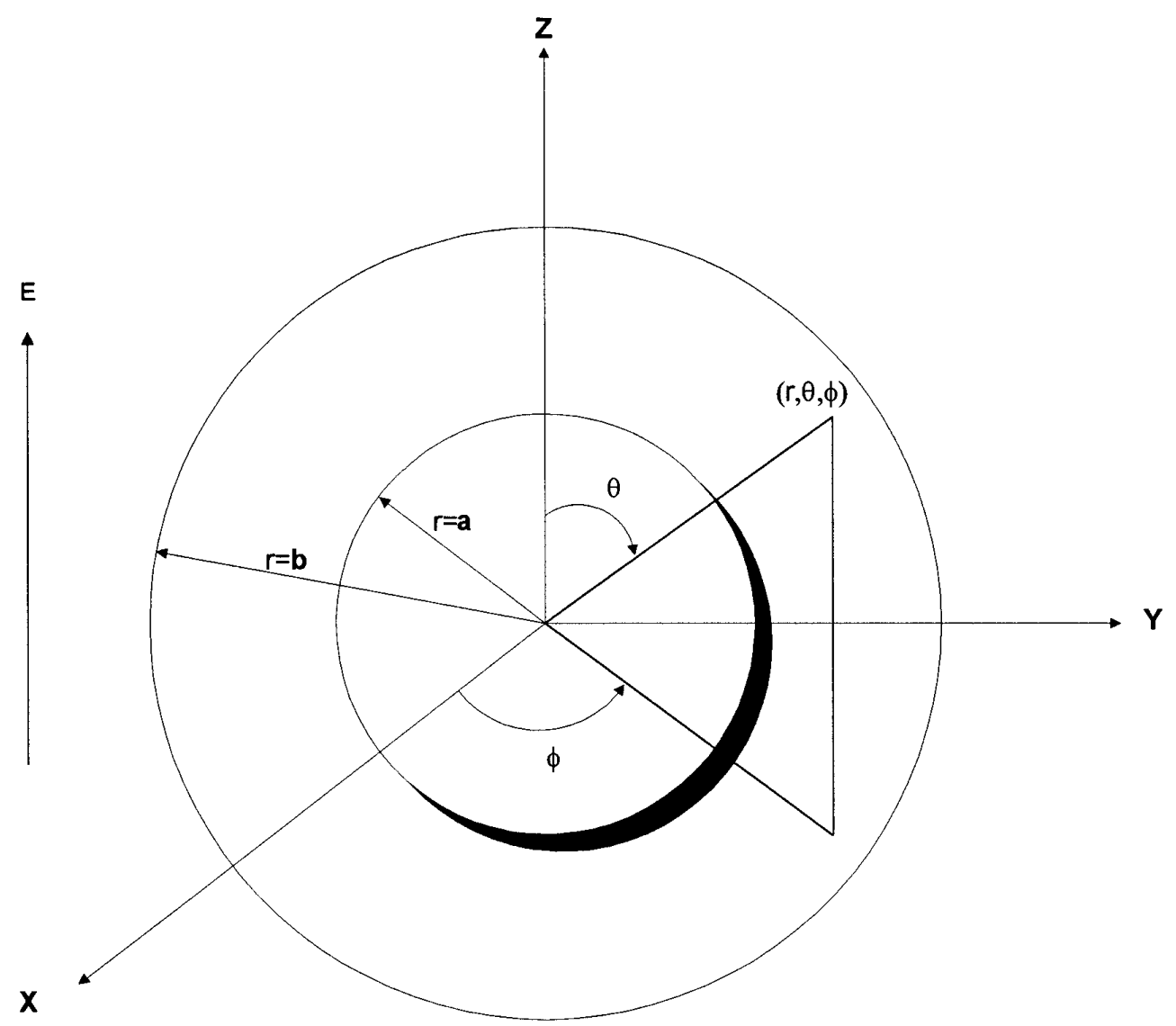

FIG. 1. Schematic representation of the system under consideration.

obtaining an analytical expression for the mobility as a function of zeta potential for a double layer of finite thickness is not an easy task. To simulate the electrophoretic behavior of a colloidal particle in a porous media, Zydney (14) considered the electrophoretic motion of a nonconductive spherical particle at the center of a spherical cavity for the case of a finite double layer. This geometry was also adopted in the analysis of a concentrated suspension (21-25). It was concluded that the boundary effect is on the order of $\lambda$ if the double layer is thick (14). This is different from that for a thin double layer where it is on the order of $\lambda^{3}$. Ennis and Anderson (26) used the method of reflections to investigate the electrophoresis of a spherical particle near a flat wall, in a slit, and in a cylindrical pore for the case of finite double layer thickness. It was assumed that the electrical potential is low, and the applied field is weak. The former implies that the Poisson-Boltzmann equation governing the variation of electrical potential can be approximated by a linear expression, which is readily solvable, and the latter implies that the effect of double layer distortion can be neglected. The analysis of Zydney (14) was extended recently by Lee et al. (27) to the case where a nonlinear Poisson-Boltzmann equation is considered. It was found that using a linearized Poisson-Boltzmann equation can lead to an appreciable deviation in the electrophoretic mobility even the electrical potential is low.

The analysis of Lee et al. (27) neglected the effect of double layer distortion, a factor which may play a significant role in the determination of the electrophoretic behavior of a particle. This effect is taken into account in the present study.

\section{THEORY}

The system under consideration is illustrated in Fig. 1. A nonconducting, spherical particle of radius $a$ is placed at the center of a spherical cavity of radius $b$, and a uniform electric field $E$ in the $z$ direction is applied. The spherical coordinates $(r, \theta, \phi)$ are adopted in the following analysis. The electrokinetic equations include the ion conservation, the electrical potential, and the fluid dynamic equations. The conservation of ions leads to

$$
\frac{\partial n_{j}}{\partial t}=\vec{\nabla} \cdot\left[D_{j}\left[\vec{\nabla} n_{j}+\frac{z_{j} e n_{j}}{k T} \vec{\nabla} \phi\right]+n_{j} \overrightarrow{\mathrm{V}}\right],
$$

where $\vec{\nabla}$ is the gradient operator, $t$ the time, $n_{j}, D_{j}$, and $z_{j}$ the 
number concentration, the diffusivity, and the valence of ion species $j$, respectively, $e$ the elementary charge, $\phi$ the electrical potential, and $\nabla$ the fluid velocity. The electrical potential is governed by the Poisson equation:

$$
\nabla^{2} \phi=-\frac{\rho}{\varepsilon}=-\sum_{j=1}^{M} \frac{z_{j} e n_{j}}{\varepsilon},
$$

where $\varepsilon$ is the permittivity of the liquid phase and $M$ the total number of ionic species.

The flow field is described by the Navier-Stokes equation under the creeping flow condition

$$
\begin{aligned}
& \vec{\nabla} \cdot \nabla=0, \\
& \rho_{\mathrm{f}} \frac{\partial \overrightarrow{\mathrm{V}}}{\partial t}=\eta \boldsymbol{\nabla}^{2} \nabla-\vec{\nabla} p-\rho \vec{\nabla} \phi
\end{aligned}
$$

where $p$ is the pressure, and $\rho_{\mathrm{f}}$ and $\eta$ the fluid density and viscosity, respectively. The last term on the right-hand side of Eq. [4] denotes the electrical body force. Here, we assume that the fluid is incompressible and has a constant viscosity. Moreover, the motion of the particle is assumed to be slow so that the system is at a quasisteady state, and the terms that involve the time derivative in the governing equations can be neglected.

Suppose that the electrical potential, $\phi$, can be decomposed into the electrical potential that would exist in the absence of the applied electric field, $\phi_{1}$, and that outside the particle arising from the applied electric field, $\phi_{2}$, that is, $\phi=\phi_{1}+\phi_{2}$. Following the same treatment as that employed by O'Brien and White (9), a perturbation function, $g_{j}$, defined below is used to describe the double layer polarization:

$$
n_{j}=n_{j 0} \exp \left(-\frac{z_{j} e\left(\phi_{1}+\phi_{2}+g_{j}\right)}{k T}\right),
$$

where $k$ and $T$ are, respectively, the Boltzmann constant and the absolute temperature, and $n_{j 0}$ the bulk concentration of ion species $j$. The pressure term in Eq. [4] can be eliminated by adopting the stream function representation (28):

$$
\vec{i}_{\phi} \mathbf{E}^{4} \psi=-\frac{1}{\eta} \sin \theta \vec{\nabla} \times\left(\rho \vec{\nabla}\left(\phi_{1}+\phi_{2}\right)\right),
$$

where $\vec{i}_{\phi}$ is the unit vector in the $\phi$ direction, $\psi$ denotes the stream function, and the operator $\mathbf{E}^{4}$ is defined as $\mathbf{E}^{4}=\mathbf{E}^{2} \mathbf{E}^{2}$ :

$$
\mathbf{E}^{2}=\frac{\partial^{2}}{\partial r^{2}}+\frac{\sin \theta}{r^{2}} \frac{\partial}{\partial \theta}\left(\frac{1}{\sin \theta} \frac{\partial}{\partial \theta}\right)
$$

The $r$ and the $\theta$ components of the velocity $\overrightarrow{\mathrm{v}}, \mathrm{v}_{r}$, and $\mathrm{v}_{\theta}$, can be described in terms of $\psi$ by

$$
\begin{aligned}
& \mathrm{v}_{r}=-\frac{1}{r^{2} \sin \theta} \frac{\partial \psi}{\partial \theta}, \\
& \mathrm{v}_{\theta}=\frac{1}{r \sin \theta} \frac{\partial \psi}{\partial r} .
\end{aligned}
$$

Let us consider the case where both the particle and the cavity are nonconductive and each has a constant surface potential characterized by $\zeta_{a}$ and $\zeta_{b}$, respectively. Then, the boundary conditions for $\phi_{1}$ are

$$
\begin{aligned}
& \phi_{1}=\zeta_{a} \text { at } r=a, \\
& \phi_{1}=\zeta_{b} \text { at } r=b .
\end{aligned}
$$

The boundary conditions for $\phi_{2}$ are assumed as

$$
\begin{aligned}
& \frac{\partial \phi_{2}}{\partial r}=0 \text { at } r=a, \\
& \frac{\partial \phi_{2}}{\partial r}=-E_{z} \cos \theta \text { at } r=b .
\end{aligned}
$$

We consider the case where the ion concentration remains at the equilibrium value on the cavity surface, and the surface of particle is ion-impenetrable. Therefore, we have

$$
\begin{aligned}
g_{j} & =-\phi_{2} \text { at } r=b, \\
-\frac{z_{j} e}{k T} \frac{\partial g_{j}}{\partial r} & =\frac{1}{D_{j}} U \text { at } r=a .
\end{aligned}
$$

Under the applied electric field, the particle moves in the $z$ direction with velocity $U$, the electrophoretic velocity. Suppose that the cavity remains fixed. These lead to the following boundary conditions for the Navier-Stokes equation:

$$
\begin{aligned}
& \mathrm{v}_{r}=U \cos \theta, \mathrm{v}_{\theta}=-U \sin \theta \text { at } r=a, \\
& \mathrm{v}_{r}=0, \mathrm{v}_{\theta}=0 \text { at } r=b,
\end{aligned}
$$

or in terms of the stream function $\psi$ as

$$
\psi=-\frac{1}{2} U r^{2} \sin \theta \text { and } \frac{\partial \psi}{\partial r}=-U r \sin ^{2} \theta \text { at } r=a,
$$

$$
\psi=\frac{\partial \psi}{\partial r}=0 \text { at } r=b
$$


Since all the dependent variables are symmetric about the $z$ axis, we have

$\frac{\partial \phi_{1}}{\partial \theta}=\frac{\partial \phi_{2}}{\partial \theta}=\frac{\partial g_{1}}{\partial \theta}=\frac{\partial g_{2}}{\partial \theta}=\psi=\frac{\partial \psi}{\partial \theta}=0$ at $\theta=0$

and at $\theta=\pi$.

Suppose that the liquid phase contains only one electrolyte $(M=2)$. Let $z_{1}$ and $z_{2}$ be, respectively, the valences of cation and anion, $\alpha=-z_{2} / z_{1}$. The electroneutrality in the bulk liquid phase implies $n_{20}=\left(n_{10} / \alpha\right)$. The reciprocal Debye length, $\kappa$, is defined as

$$
\kappa=\left[\sum_{j=1}^{2} n_{j 0}\left(e z_{j}\right)^{2} / \varepsilon k T\right]^{1 / 2}
$$

It can be shown that

$$
n_{10} z_{1}=\frac{(\kappa a)^{2} \varepsilon k T}{(1+\alpha) e^{2} a^{2} z_{1}} .
$$

Define $U_{E}=\left(\varepsilon \zeta_{k}^{2} / \eta a\right)$, which is the velocity of a particle predicted by the Smoluchowski's theory when an electric field of strength $\left(\zeta_{k} / a\right)$ is applied, where $\zeta_{k}$ is $\zeta_{a}$ if $\zeta_{a} \neq 0$ and is $\zeta_{b}$ if $\zeta_{a}=0$.

To simplify the mathematical manipulation, the governing equations are rewritten in a scaled form. In the discussion below, a symbol with an asterisk denotes a scaled quantity. Suppose that the applied electric field is weak so that the expressions for the distortion of double layer, the electric potential, and the flow field near a particle can be linearized. For example, the scaled number concentrations of ions, $n_{1}^{*}$ and $n_{2}^{*}$, can be approximated by

$$
\begin{aligned}
& n_{1}^{*}=\exp \left(-\phi_{r} \phi_{1}^{*}\right)\left[1-\phi_{r}\left(\phi_{2}^{*}+g_{1}^{*}\right)\right], \\
& n_{2}^{*}=\exp \left(\alpha \phi_{r} \phi_{1}^{*}\right)\left[1+\alpha \phi_{r}\left(\phi_{2}^{*}+g_{2}^{*}\right)\right],
\end{aligned}
$$

where $g_{j}^{*}=g_{j} / \zeta_{k}, j=1,2, \phi_{1}^{*}=\phi_{1} / \zeta_{k}, \phi_{2}^{*}=\phi_{2} / \zeta_{k}$, and $\phi_{r}=\zeta_{k} z_{1} e / k T$, where $\zeta_{k}$ is $\zeta_{a}$, if $\zeta_{a} \neq 0$, and is $\zeta_{b}$ if $\zeta_{a}=0$. The electrokinetic equations can be linearized by neglecting the terms that involve products of small quantities such as, $g_{1}^{*}$, $g_{2}^{*}$, and $\phi_{2}^{*}$. For instance, the variation of $\phi_{1}^{*}$ becomes

$$
\nabla^{* 2} \phi_{1}^{*}=-\frac{1}{(1+\alpha)} \frac{(\kappa a)^{2}}{\phi_{r}}\left[\exp \left(-\phi_{r} \phi_{1}\right)-\exp \left(\alpha \phi_{r} \phi_{1}\right)\right]
$$

If the particle is charged and the cavity uncharged, the boundary conditions associated with Eq. [17] are

$$
\phi_{1}^{*}=1 \text { at } r^{*}=1 \text {, and } \phi_{1}^{*}=\zeta_{b} / \zeta_{a} \text { at } r^{*}=\frac{1}{\lambda},
$$

where $r^{*}=r / a$, and $\lambda=a / b$. If the particle is uncharged and the cavity charged, then the boundary conditions associated with Eq. [17] are

$$
\phi_{1}^{*}=0 \text { at } r^{*}=1 \text {, and } \phi_{1}^{*}=1 \text { at } r^{*}=1 / \lambda .
$$

Similarly, the variation of $\phi_{2}^{*}$ is described by

$$
\begin{gathered}
\boldsymbol{\nabla}^{* 2} \phi_{2}^{*}-\frac{(\kappa a)^{2}}{(1+\alpha)}\left[\exp \left(-\phi_{r} \phi_{1}^{*}\right)+\alpha \exp \left(\alpha \phi_{r} \phi_{1}^{*}\right)\right] \phi_{2}^{*} \\
=\frac{(\kappa a)^{2}}{(1+\alpha)}\left[\exp \left(-\phi_{r} \phi_{1}^{*}\right) g_{1}^{*}+\exp \left(\alpha \phi_{r} \phi_{1}^{*}\right) \alpha g_{2}^{*}\right]
\end{gathered}
$$

The associated boundary conditions are

$$
\frac{\partial \phi_{2}^{*}}{\partial r}=0 \text { at } r^{*}=1 \text { and } \frac{\partial \phi_{2}^{*}}{\partial r}=-E_{z}^{*} \cos \theta \text { at } r^{*}=\frac{1}{\lambda},
$$

where $E_{z}^{*}=E_{z} a / \zeta_{a}$. The variation of $g_{j}^{*}$ is described by

$$
\begin{gathered}
\boldsymbol{\nabla}^{* 2} g_{1}^{*}-\phi_{r} \vec{\nabla} * \phi_{1}^{*} \cdot \vec{\nabla} * g_{1}^{*}=\phi_{r}^{2} \mathrm{Pe}_{1} \vec{\nabla} * \vec{\nabla} * \phi_{1}^{*}, \\
\boldsymbol{\nabla}^{* 2} g_{2}^{*}+\alpha \phi_{r} \vec{\nabla} * \phi_{1}^{*} \cdot \vec{\nabla} * g_{2}^{*}=\phi_{r}^{2} \mathrm{Pe}_{2} \nabla^{*} \cdot \vec{\nabla} * \phi_{1}^{*},
\end{gathered}
$$

where $\mathrm{Pe}_{j}=\varepsilon\left(Z_{1} e / k T\right)^{2} / \eta D_{j}, j=1,2$, is the electric Peclet number of ion species $j$, and $U^{*}=U / U_{E}$. The associated boundary conditions are

$$
\begin{aligned}
& \frac{\partial g_{1}^{*}}{\partial r^{*}}=-\mathrm{Pe}_{1} \phi_{r} U^{*} \cos \theta \text { at } r^{*}=1, \text { and } g_{1}^{*}=-\phi_{2}^{*} \text { at } r^{*}=1 / \lambda, \\
& \frac{\partial g_{2}^{*}}{\partial r^{*}}=\frac{\mathrm{Pe}_{2} \phi_{r}}{\alpha} U^{*} \cos \theta \text { at } r^{*}=1, g_{2}^{*}=-\phi_{2}^{*} \text { at } r^{*}=\frac{1}{\lambda}
\end{aligned}
$$

The scaled Navier-Stokes equation is

$$
\mathbf{E}^{* 4} \psi^{*}=\frac{(\kappa a)^{2}}{(1+\alpha)}\left[\left(\frac{\partial g_{1}^{*}}{\partial \theta} n_{1}^{*}+\frac{\partial g_{2}^{*}}{\partial \theta} \alpha n_{2}^{*}\right) \frac{\partial \phi_{1}^{*}}{\partial r^{*}}\right] \sin \theta .
$$

The associated boundary conditions are

$$
\begin{aligned}
\psi^{*} & =-\frac{1}{2} U^{*} r^{* 2} \sin \theta \text { at } r^{*}=1, \\
\frac{\partial \psi^{*}}{\partial r^{*}} & =-U^{*} r^{*} \sin ^{2} \theta \text { at } r^{*}=1 \\
\psi^{*} & =\frac{\partial \psi^{*}}{\partial r^{*}}=0 \text { at } r^{*}=\frac{1}{\lambda}
\end{aligned}
$$

It can be shown that each of the functions $\phi_{2}^{*}, g_{1}^{*}, g_{2}^{*}$, and $\psi^{*}$ 
can be expressed as the product of a radial function and an angular function, and the solutions to Eqs. [17]-[21] subject to Eqs. [17a]-[21c] take the form

$$
\begin{aligned}
& \phi_{2}^{*}=\Phi_{2}(r) \cos \theta \\
& g_{1}^{*}=G_{1}(r) \cos \theta \\
& g_{2}^{*}=G_{2}(r) \cos \theta \\
& \psi^{*}=\Psi(r) \sin ^{2} \theta
\end{aligned}
$$

On the basis of these expressions, the original problem becomes one-dimensional. Equation [17] reduces to

$$
\mathbf{L} \phi_{1}^{*}=-\frac{1}{(1+\alpha)} \frac{(\kappa a)^{2}}{\phi_{r}}\left[\exp \left(-\phi_{r} \phi_{1}\right)-\exp \left(\alpha \phi_{r} \phi_{1}\right)\right]
$$

where the operator $\mathbf{L}$ is defined by

$$
\mathbf{L} \equiv \frac{d^{2}}{d r^{* 2}}+\frac{2}{r^{*}} \frac{d}{d r^{*}}-\frac{2}{r^{* 2}}
$$

The associated boundary conditions are, if the particle is charged and cavity uncharged,

$$
\begin{aligned}
& \phi_{1}^{*}=1 \text { at } r^{*}=1, \\
& \phi_{1}^{*}=\zeta_{b} / \zeta_{a} \text { at } r^{*}=\frac{1}{\lambda} .
\end{aligned}
$$

If the particle is uncharged and cavity charged, the boundary conditions associated with Eq. [26] are

$$
\begin{gathered}
\phi_{1}^{*}=0 \text { at } r^{*}=1, \\
\phi_{1}^{*}=1 \text { at } r^{*}=\frac{1}{\lambda} .
\end{gathered}
$$

Similarly, Eqs. [18]-[21] reduce respectively to

$$
\begin{gathered}
{\left[\mathbf{L}-\frac{(\kappa a)^{2}}{1+\alpha}\left[\exp \left(-\phi_{r} \phi_{1}^{*}\right)+\alpha \exp \left(\alpha \phi_{r} \phi_{1}^{*}\right)\right]\right] \Phi_{2}} \\
=\frac{(\kappa a)^{2}}{1+\alpha}\left[G_{1} \exp \left(-\phi_{r} \phi_{1}^{*}\right)+\alpha G_{2} \exp \left(\alpha \phi_{r} \Phi_{1}\right)\right], \\
\mathbf{L} G_{1}-\phi_{r} \frac{d \phi_{1}^{*}}{d r^{*}} \frac{d G_{1}}{d r^{*}}=\mathrm{Pe}_{1} \mathrm{~V}_{r}^{*} \frac{d \phi_{1}^{*}}{d r^{*}}, \\
\mathbf{L} G_{2}+\alpha \phi_{r} \frac{d \phi_{1}^{*}}{d r^{*}} \frac{d G_{2}}{d r^{*}}=\mathrm{Pe}_{2} \mathrm{~V}_{r}^{*} \frac{d \phi_{1}^{*}}{d r^{*}}
\end{gathered}
$$

and

$$
\mathbf{D}^{4} \Psi=-\frac{(\kappa a)^{2}}{1+\alpha}\left[\left(n_{1}^{*} G_{1}+\alpha n_{2}^{*} G_{2}\right) \frac{d \phi_{1}^{*}}{d r^{*}}\right],
$$

where the operator $\mathbf{D}^{4}$ is defined as $\mathbf{D}^{4}=\mathbf{D}^{2} \mathbf{D}^{2}$ with

$$
\mathbf{D}^{2}=\frac{d^{2}}{d r^{* 2}}-\frac{2}{r^{* 2}}
$$

The associated boundary conditions are:

$$
\begin{aligned}
\frac{d \Phi_{2}}{d r^{*}} & =0 \text { at } r^{*}=1, \\
\frac{d \Phi_{2}}{d r^{*}} & =-E_{z}^{*} \text { at } r^{*}=\frac{1}{\lambda}, \\
\frac{d G_{j}}{d r^{*}} & =-\frac{P e_{j}}{\phi_{r}} U^{*} \text { at } r^{*}=1, j=1,2, \\
G_{j} & =-\Phi_{2} \text { at } r^{*}=1 / \lambda, j=1,2, \\
\Psi & =-\frac{1}{2} U^{*} r^{* 2} \text { and } \frac{d \Psi}{d r^{*}}=-U^{*} r^{*} \text { at } r^{*}=1, \\
\Psi & =\frac{d \Psi}{d r^{*}}=0 \text { at } r^{*}=\frac{1}{\lambda} .
\end{aligned}
$$

The mobility of a particle can be estimated on the fact that the sum of the external forces acting on it in the $z$ direction which includes the electric force, $F_{E}$, and the hydrodynamic forces, $F_{D_{z}}$, vanishes at the steady state, that is,

$$
F_{D_{z}}+F_{E_{z}}=0
$$

The electric force can be calculated by

$$
F_{E_{z}}=\iint \sigma(-\vec{\nabla} \phi) d \vec{A}
$$

where $\sigma$ is the surface charge density which is related to $\phi_{1}$ by Gauss's divergence theorem. It can be shown that

$$
F_{E_{z}}=\frac{8}{3} \pi \varepsilon \zeta_{a}^{2}\left(r^{*} \frac{d \phi_{1}^{*}}{d r^{*}} \Phi_{2}\right)_{r^{*}=1}=F_{E_{z}}^{*} \pi \varepsilon \zeta_{a}^{2},
$$

where

$$
F_{E_{z}}^{*}=\frac{8}{3}\left(r^{*} \frac{d \phi_{1}^{*}}{d r^{*}} \Phi_{2}\right)_{r^{*}=1}
$$


In spherical coordinates, $F_{D_{z}}$ can be evaluated by (27)

$$
\begin{aligned}
F_{D_{z}}=\eta \pi \int_{0}^{\pi}\left(r^{4} \sin ^{3} \theta \frac{\partial}{\partial r} \frac{E^{2} \psi}{r^{2} \sin ^{2} \theta}\right)_{r=a} d \theta & \\
& \quad-\pi \int_{0}^{\pi}\left(r^{2} \sin ^{2} \theta \rho \frac{\partial \phi}{\partial \theta}\right)_{r=a} d \theta .
\end{aligned}
$$

On the basis of the linearized formulation adopted in the present study, this expression becomes, in terms of scaled quantities,

$$
\begin{aligned}
& F_{D_{z}}=\frac{4}{3} \pi \varepsilon \zeta_{a}^{2}\left(r^{* 4} \frac{\partial}{\partial r^{*}}\left(\frac{D^{2} \Psi}{r^{* 2}}\right)\right)_{r^{*}=1} \\
&+\frac{4}{3} \pi \varepsilon \zeta_{a}^{2} \frac{(\kappa a)^{2}}{(1+\alpha a) \phi_{r}}\left[r ^ { * 2 } \left[\exp \left(-\phi_{r} \phi_{1}^{*}\right)\right.\right. \\
&\left.\left.-\exp \left(\alpha \phi_{r} \phi_{1}^{*}\right)\right] \Phi_{2}\right]_{r^{*}=1} .
\end{aligned}
$$

For convenience, this expression is rewritten as

$$
F_{D_{z}}^{*}=F_{D_{f z}}^{*}+F_{D_{e z}}^{*},
$$

where

$F_{D_{z}}^{*}=\frac{F_{D_{z}}}{\pi \varepsilon \zeta_{a}^{2}}$

$F_{D_{f^{2}}}^{*}=\frac{4}{3}\left(r^{*^{4}} \frac{\partial}{\partial r^{*}}\left(\frac{D^{2} \Psi}{r^{* 2}}\right)\right)_{r^{*}=1}$,

$$
\begin{aligned}
F_{D_{e z}}^{*}=\frac{4}{3} \frac{(\kappa a)^{2}}{(1+\alpha a) \phi_{r}}\left[r ^ { * 2 } \left[\exp \left(-\phi_{r} \phi_{1}^{*}\right)\right.\right. & \\
& \left.\left.-\exp \left(\alpha \phi_{r} \phi_{1}^{*}\right)\right] \Phi_{2}\right]_{r^{*}=1} .
\end{aligned}
$$

In Eq. [37], $F_{D_{f}}^{*}$ and $F_{D_{e z}}^{*}$ arise from, respectively, the viscous and the electric body forces exert on the particle. According to O'Brien and White (9), the problem of solving the linearized governing equations, Eqs. [26]-[31f], can be decomposed into two subproblems. The first one involves the solution of the problem that a particle moves at a uniform velocity $U$ in the absence of the applied field. The boundary conditions associated with this problem are

$$
\begin{aligned}
& u_{r}^{*}=U^{*} \cos \theta, \text { and } u_{\theta}^{*}=-U^{*} \sin \theta \text { at } r^{*}=1, \\
& \phi_{2}^{*}=0 \text { at } r^{*}=\frac{1}{\lambda} .
\end{aligned}
$$

In the second problem, the particle is fixed in the applied electric field, and a no-slip condition is assumed at cavity surface. The boundary conditions are

$$
\begin{gathered}
u_{r}^{*}=u_{\theta}^{*}=0 \text { at } r^{*}=1, \\
\frac{\partial \phi_{2}^{*}}{\partial r^{*}}=-E^{*} \cos \theta \text { at } r^{*}=\frac{1}{\lambda} .
\end{gathered}
$$

The force required to move the particle in the first problem, $f_{1}$, is proportional to $U^{*}$, that is, $f_{1}=\delta U^{*}$. Similarly, the force exerts on the particle in the second problem, $f_{2}$, is proportional to $E^{*}$, that is, $f_{2}=\beta E^{*}$. The fact that the sum of the external forces acting on the particle vanishes leads to $U_{\mathrm{m}}^{*}=-\beta / \delta, U_{\mathrm{m}}^{*}$ being the scaled mobility of the particle. Note that choosing the scaling factor $U_{E}$ for the electrophoretic velocity $U$ has the advantage of simplifying Eq. [21] so that the unknown terminal velocity of the particle will not present. The scaled mobility $U_{\mathrm{m}}^{*}$ thus obtained is the same as that of Zydney (14). The mobility defined by O'Brian and White (9) can be expressed as $6 \pi \phi_{r} U_{m}^{*}$

The set of differential equations, Eqs. [26]-[30] are solved by a pseudospectral method (29) based on the Chebyshev polynomials. For the problem under consideration, this method is readily applicable and it has several desirable properties, such as a fast rate of convergence and the convergent properties independent of the associated boundary conditions. Also, the mini-max property typically associated with the Chebyshev polynomial is maintained. In our case, the computational domain is one-dimensional, and only the variations of the dependent variables in the $r$ direction need to be determined. Here we assume that an unknown function $f(r)$ can be expressed in an $N$ th-order approximation:

$$
f_{N}(r)=\sum_{i=0}^{N} f\left(r_{i}\right) h_{i}(r),
$$

where $f\left(r_{i}\right)$ is the value of $f(r)$ at the $i$ th collocation point. The interpolation polynomial, $h_{i}(r)$, is a function of the collocation points which are determined by mapping the computational domain of the radial variable $r$ onto the interval of $y,[-1,1]$, by

$$
r=\frac{b-a}{2} y+\frac{b+a}{2}
$$

The $(N+1)$ interpolation points in the interval $[-1,1]$ are chosen to be the extrema of an Nth-order Chebyshev polynomial, $T_{N}(y)$, and

$$
y_{j}=\cos \left(\frac{\pi j}{N}\right), j=0,1, \ldots, N
$$


The corresponding interpolation polynomial $h_{j}(y)$ is

$$
\begin{array}{r}
h_{j}(y)=\left[(-1)^{j+1}\left(1-y^{2}\right) \frac{d T_{N}(y)}{d y}\right] /\left[c_{j} N^{2}\left(y-y_{j}\right)\right], \\
j=0,1, \ldots, N,
\end{array}
$$

where $c_{j}$ is defined by

$$
c_{j}= \begin{cases}2, & j=0, N . \\ 1, & 1 \leq j \leq N-1 .\end{cases}
$$

Differentiating Eq. [40] with respect to $r$ yields

$$
\frac{d f_{N}(r)}{d r}=\sum_{i=0}^{N} \frac{d h_{i}(r)}{d r} f_{N}\left(r_{i}\right)=\sum_{i=0}^{N} \sum_{j=0}^{N}\left(D_{N}\right)_{i j} f_{i}\left(x_{j}\right),
$$

where $\left(D_{N}\right)_{i j}$ denotes the $i j$ th element of the derivative matrix which can be obtained by using the method proposed by Gottlieb (28). The governing equations, Eqs. [26]-[30], are rewritten in a discretized form. The discretized Eq. [26] is first solved by adopting a Newton-Raphson iteration scheme to obtain $\phi_{1}^{*}$ first, and the rest of the discretized governing equations are then solved. Although the latter is a set of linear algebraic equations, the solution procedure still involves an iterative procedure since they are coupled. The independence of the solution on the number of grids is checked to ensure that the mesh used is fine enough, and the solution obtained is correct. Sixtyfive nodal points are used in the numerical procedure. Double precision is used throughout the numerical computation.

\section{DISCUSSION}

Two cases are considered in the numerical simulations: (a) the particle is positively charged and the cavity uncharged, and (b) the particle is uncharged and the cavity positively charged.

\subsection{Particle Positively Charged, Cavity U ncharged}

Figure $2 \mathrm{a}$ shows the variation of the scaled mobility of a particle, $U_{\mathrm{m}}^{*}$, as a function of $\kappa a$ at various scaled surface potential of particle, $\phi_{r}\left(=\zeta_{a} z_{1} e / k T\right)$, for the case where the particle is positively charged and the cavity uncharged. The variation of the absolute percentage deviation of the mobility of a particle as a function of $\kappa a$ at various $\phi_{r}$ is presented in Fig. 2b. The percentage deviation is defined by $\mid\left(U_{\mathrm{m}}^{*}-U_{\mathrm{L}}^{*}\right) /$ $\mathrm{U}_{\mathrm{m}}^{*} \mid \times 100 \%, U_{\mathrm{L}}^{*}$ being the scaled mobility based on the model where the linearized Poisson-Boltzmann equation is considered and the double layer distortion neglected (14). Figure 2a and $\mathrm{b}$ reveal that, in general, the higher the $\phi_{r}$, the greater the deviation of the result based on the linearized Poisson-Boltzmann equation from the present result, as expected. As can be
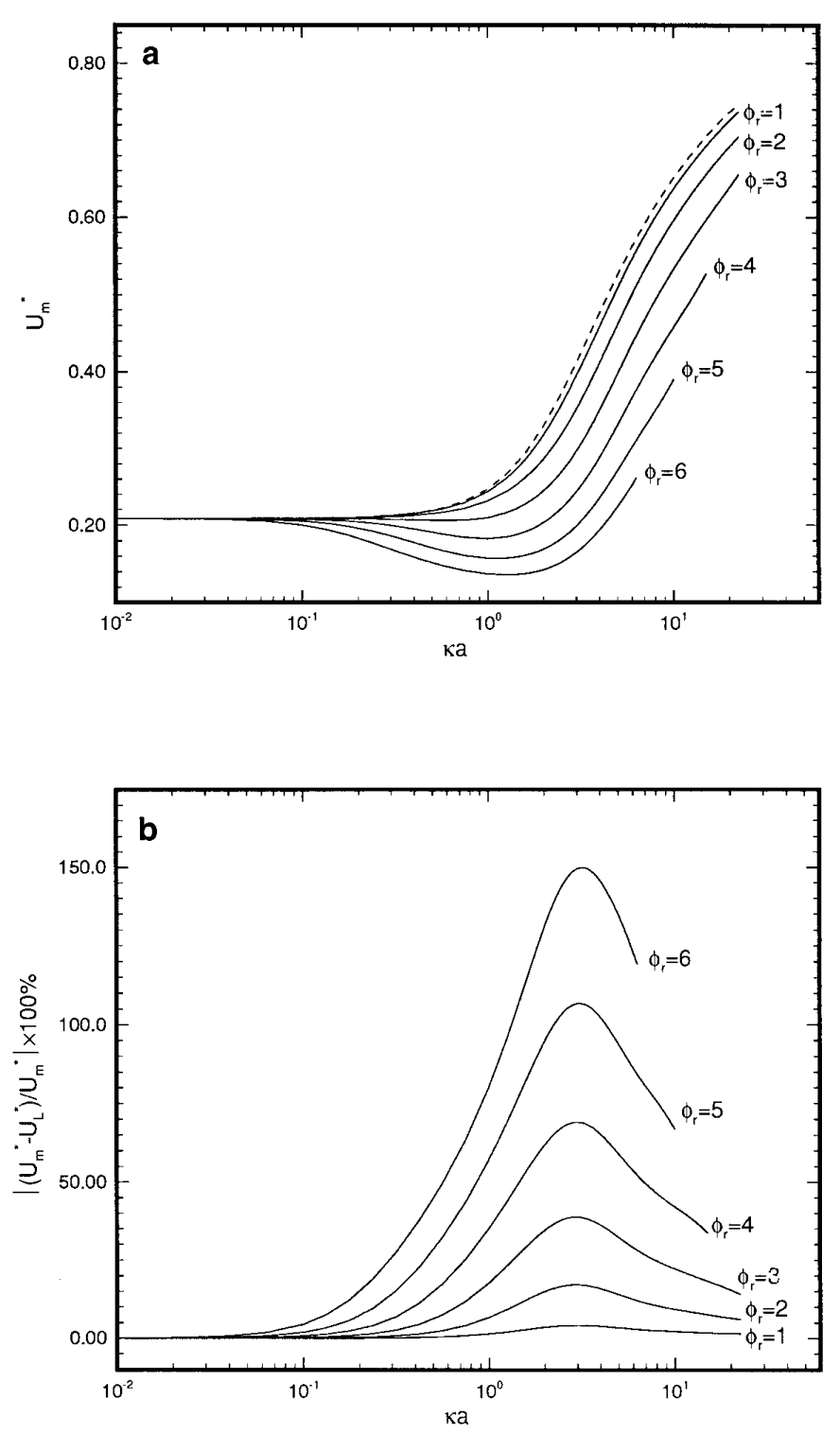

FIG. 2. (a) Variation of the scaled mobility of a particle, $U_{\mathrm{m}}^{*}$, as a function of $\kappa a$ at various scaled surface potential of particle, $\phi_{r}$, for the case where the particle is positively charged and the cavity is uncharged. The dashed line represents the result based on the linearized Poisson-Boltzmann equation. (b) Variation of the absolute percentage deviation of the mobility of a particle as a function of $\kappa a$ at various $\phi_{r} . U_{\mathrm{L}}^{*}$ is the result based on (14). Key: $\lambda=0.5$, $\mathrm{Pe}_{1}=\mathrm{Pe}_{2}=0.1$, and $\alpha=1$.

seen from Fig. $2 b$, this deviation vanishes, however, if $\kappa a$ is either very large or very small. In other words, the result of Zydney (14) can be recovered as the limiting case of the present study. Figure 2a shows that for a low to medium $\phi_{r}$, $U_{\mathrm{m}}^{*}$ increases with $\kappa a$. If $\phi_{r}$ is high, the variation of $U_{\mathrm{m}}^{*}$ as a function of $\kappa a$ has a minimum. This was also observed by Wiersema (8) and O'Brien (9) for the electrophoresis of a spherical particle in an infinite fluid. The rationale behind this can be elaborated as following. According to the theoretical deviation, the mobility of a particle can be expressed as the 
ratio $-\beta / \delta$. The numerator is the force exerts on the particle, which is fixed at the center of the cavity, per unit electric field, and the denominator that per unit velocity of particle in the absence of electric field. These forces are determined by the sum of $F_{E_{z}}$ and $F_{D_{z}}$. Therefore, both $\delta$ and $\beta$ are related to double layer distortion and are functions of $\Phi_{2}, G_{1}$, and $G_{2}$, as suggested by Eqs. [34], [36], and [37]. Due to the linearization of governing equations, however, $\delta$ and $\beta$ are independent of $E^{*}$ and $U^{*}$. According to Eq. [34], $F_{E_{z}}^{*}$ is determined by the product $\left(d \phi_{1}^{*} / d r^{*}\right) \Phi_{2}$. The value of $\left(d \phi_{1}^{*} / d r^{*}\right)$, or the surface charge density, increases with $\kappa a$. However, since the electric field in the double layer increases with $\kappa a$ also, the effect of the applied electric field is lessened, and $\Phi_{2}$ decreases. The higher the $\phi_{r}$, the more significant this effect is. Therefore, the variations of $F_{E_{z}}^{*}$ and the resultant $-\beta$ as a function of $\kappa a$ has a minimum at a higher $\phi_{r}$. This can be justified by the fact that the qualitative behaviors of $F_{E_{z}}^{*}$ and $-\beta$ follow the same trend, as shown in Fig. 3a and b. The minimum does not appear if the effect of double layer distortion is neglected. Note that $U_{\mathrm{m}}^{*}$ is independent of $\phi_{r}$ if $\kappa a$ is fixed in Zydney's result (14).

Figure 4 shows the variation of the scaled mobility $U_{\mathrm{m}}^{*}$ as a function of $\kappa a$ at various $\lambda$ (= radius of particle/radius of cavity). As can be seen from this figure, the variation of $U_{\mathrm{m}}^{*}$ has a minimum if $\lambda$ has a medium value; it becomes inappreciable if $\lambda$ is small or large. According to Zydney (14), if $\kappa a$ is small, the effect of the spherical boundary on the mobility of a spherical particle is on the order $\lambda$, and on the order $\lambda^{3}$, if $\kappa a$ is large. This implies that the result for an isolated particle can be deduced from the present system at a small $\lambda$. The curve corresponding to $\lambda=0.1$ in Fig. 4 , for example, is approximate to the variation of the scaled electrophoretic mobility of an isolated spherical particle as a function of $\kappa a$.

The variation of $\phi_{r c}$, the scaled surface potential of particle at which the mobility has the minimum, as a function of $\lambda$ is presented in Fig. 5a, and the variation of $\kappa a_{c}$, the value of $\kappa a$ at which the mobility has the minimum, as a function of $\phi_{r}$ at various $\lambda$ shown in Fig. 5b. If $\lambda$ is small, the distance between the particle and the cavity is large. As a result, the decrease in $\Phi_{2}$ due to the electric field induced in the double layer around the particle is lessened, and, therefore, it is relatively difficult for $U_{\mathrm{m}}^{*}$ to have a minimum. On the other hand, if $\lambda$ is large, the motion of the particle is confined in a limited space, and it is also difficult for $U_{\mathrm{m}}^{*}$ to have a minimum. This is why the variation of $\phi_{r c}$ as a function of $\lambda$ has a minimum, as shown in Fig. 5a. Figure 5b suggests that, if $\lambda$ is large, the variation of $\kappa a_{c}$ as a function of $\phi_{r}$ may have a maximum. Whether this maximum appears depends largely on the relative rate of variations of $\left(d \phi_{1}^{*} / d r^{*}\right)$ and $\Phi_{2}$ as a function of $\kappa a$.

\subsection{Particle U ncharged, Cavity Positively Charged}

Figure 6(a) shows the variation of the scaled mobility of a particle, $U_{\mathrm{m}}^{*}$, as a function of $\kappa a$ at various scaled surface potential of cavity, $\phi_{r}\left(=\zeta_{b} z_{1} e / k T\right)$, and that at various $\lambda$
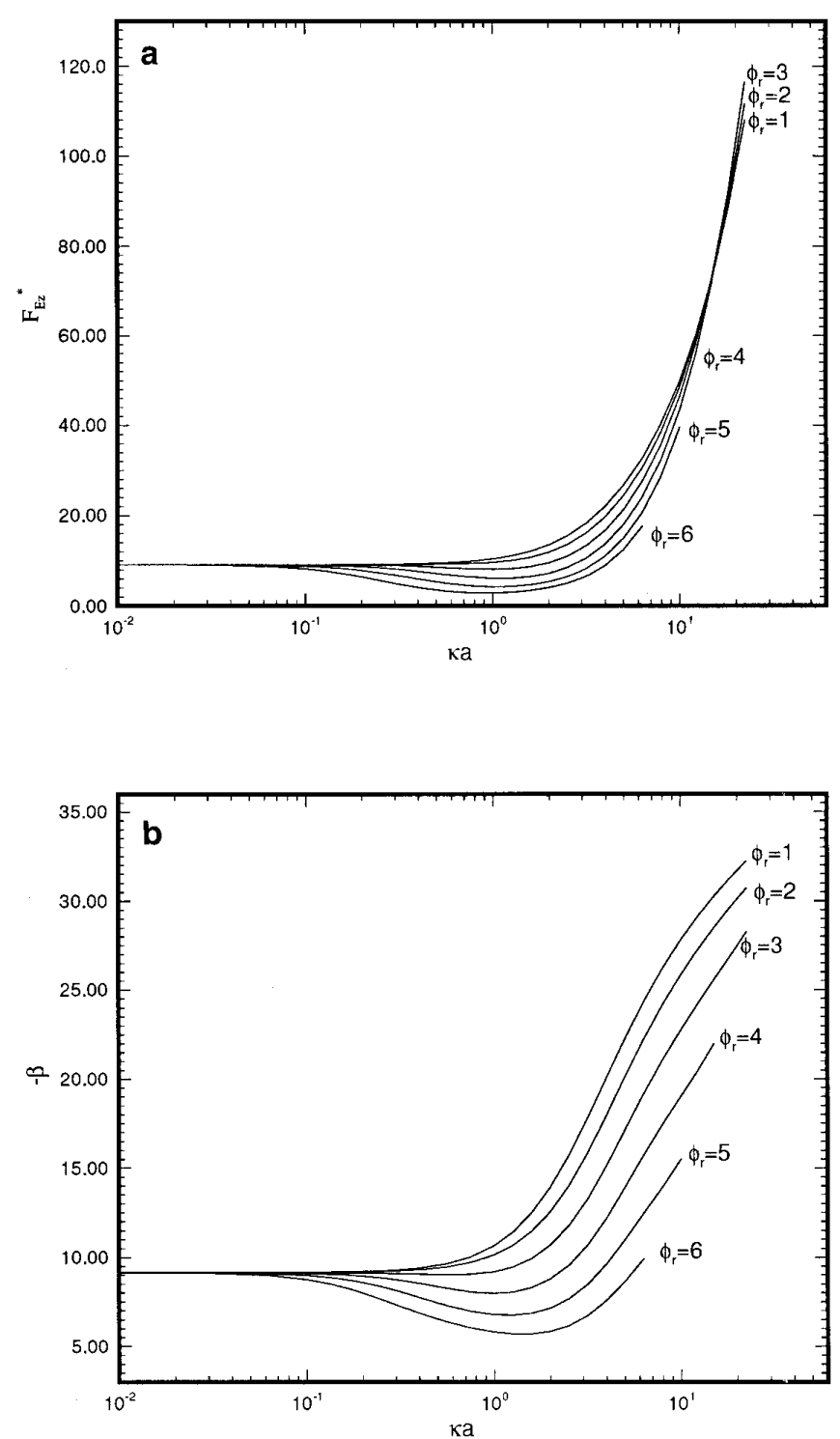

FIG. 3. (a) Variation of $F_{E z}$ as a function of $\kappa a$ at various scaled surface potential of particle, $\phi_{r}$, for the case where the particle is positively charged and the cavity is uncharged. (b) Variation of $-\beta$ as a function of $\kappa a$ at various $\phi_{r} . U_{\mathrm{m}}^{*}$ and $U_{\mathrm{L}}^{*}$ are, respectively, the scaled mobility based on the present study and that on (14). Key: same as Fig. 2.

shown in Fig. $6 b$ for the case where the particle is uncharged and the cavity positively charged. For comparison, the result based on the linearized Poisson-Boltzmann equation and neglecting the effect of double layer distortion (14) is also presented in Fig. 6a. According to Zydney (14), if $\kappa a \rightarrow 0$, negative charges will be induced on particle surface due to the presence of the positively charged cavity. Therefore, the particle will move in the $-z$ direction, that is, the mobility is negative. As $\kappa a$ increases, the double layer near the cavity surface becomes thinner, and the amount of induced negative charges on particle surface decreases. The concentrated anions in the double layer near cavity surface leads to an electroos- 
motic flow under the influence of the applied electric field. A clockwise vortex flow will appear, and the particle experiences a drag force in the $z$ direction. Therefore, the mobility of the particle may become positive as $\kappa a$ increases, and approaches the limiting result as that reported by Zydney (14) as $\kappa a \rightarrow \infty$. In our case, the double layer distortion makes the problem more complex. As revealed by Fig. 6a, for a higher $\phi_{r}$, the variation of $U_{\mathrm{m}}^{*}$ as a function of $\kappa a$ has a maximum, and if $\phi_{r}$ is low, $U_{\mathrm{m}}^{*}$ increases monotonically with $\kappa a$. Figure $6 \mathrm{~b}$ suggests that for a small to medium $\lambda$ the variation of $U_{\mathrm{m}}^{*}$ as a function of $\kappa a$ has a maximum. Due to the limitation of the available space for particle movement, the maximum mobility is relatively difficult to achieve if $\lambda$ is large.

The variations of $F_{D_{f z}}^{*}$ and $F_{E_{z}}^{*}$ as a function of $\kappa a$ at various scaled surface potential of cavity $\phi_{r}$ are illustrated in Fig. 7. In contrast to the case where the particle is charged and the cavity uncharged, the behavior of the mobility is mainly determined by one of the scaled components of the drag force $F_{D_{z}}^{*}, F_{D_{f}}^{*}$, defined in [37b], rather than by the electric force $F_{E_{z}}^{*}$. As suggested by Fig. $7 \mathrm{a}, F_{D_{f z}}^{*} \rightarrow 0$ as $\kappa a$ $\rightarrow 0$. As $\kappa a$ increases, the strength of the electroosmosic flow increases, so does $F_{D_{f}}^{*}$. However, the variation of $F_{D_{f z}}^{*}$ as a function of $\kappa a$ has a maximum if $\phi_{r}$ is high. Since the strength of the electroosmotic flow is determined by $\left(d \phi_{1}^{*} /\right.$ $\left.d r^{*}\right)$ and $G_{j}, j=1,2$, as suggested by Eq. [30], and $G_{j}$ is related to $\Phi_{2}$, as suggested by Eq. [31d], the existence of this maximum is a function of $\Phi_{2}$, which is due to the presence of the applied electric field. As can be seen from Fig. $7 \mathrm{~b}$, if $\phi_{r}$ is high, the variation of $F_{E_{z}}^{*}$ as a function $\kappa a$ has a minimum. This is because that $\left(d \phi_{1}^{*} / d r^{*}\right)_{r^{*}=1}$ decreases with an increase in $\kappa a$, and the variation of $\Phi_{2}$ as a function of $\kappa a$ has a minimum (note $\Phi_{2}$ is negative). In other words, the influence due to the applied electric field, measured by $\Phi_{2}$, has a minimum as $\kappa a$ varies. This has an indirect

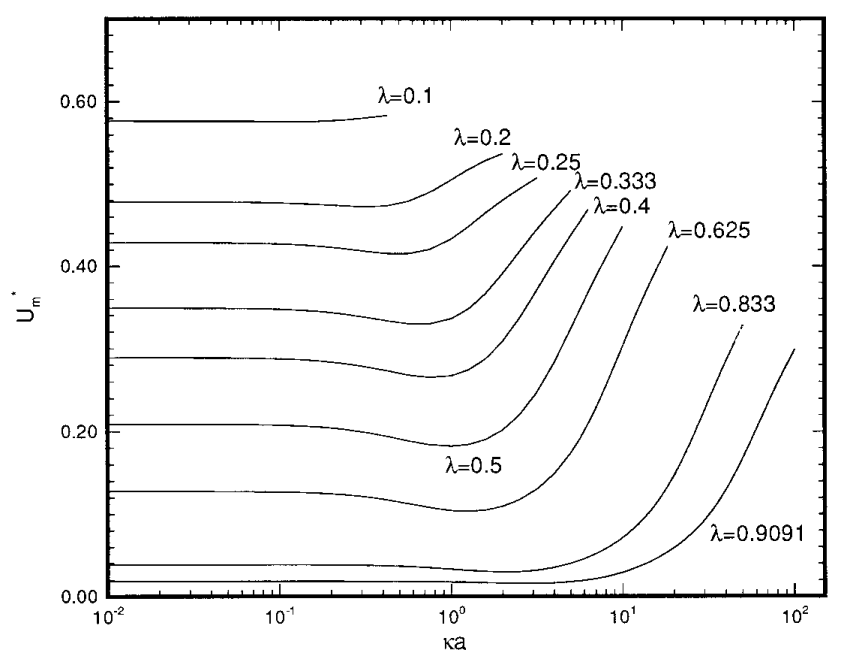

FIG . 4. Variation of the scaled mobility $U_{\mathrm{m}}^{*}$ as a function of $\kappa a$ at various $\lambda$ (= radius of particle/radius of cavity) for the case where $\phi_{r}=4$. The particle is positively charged, and the cavity is uncharged. Key: same as Fig. 2.
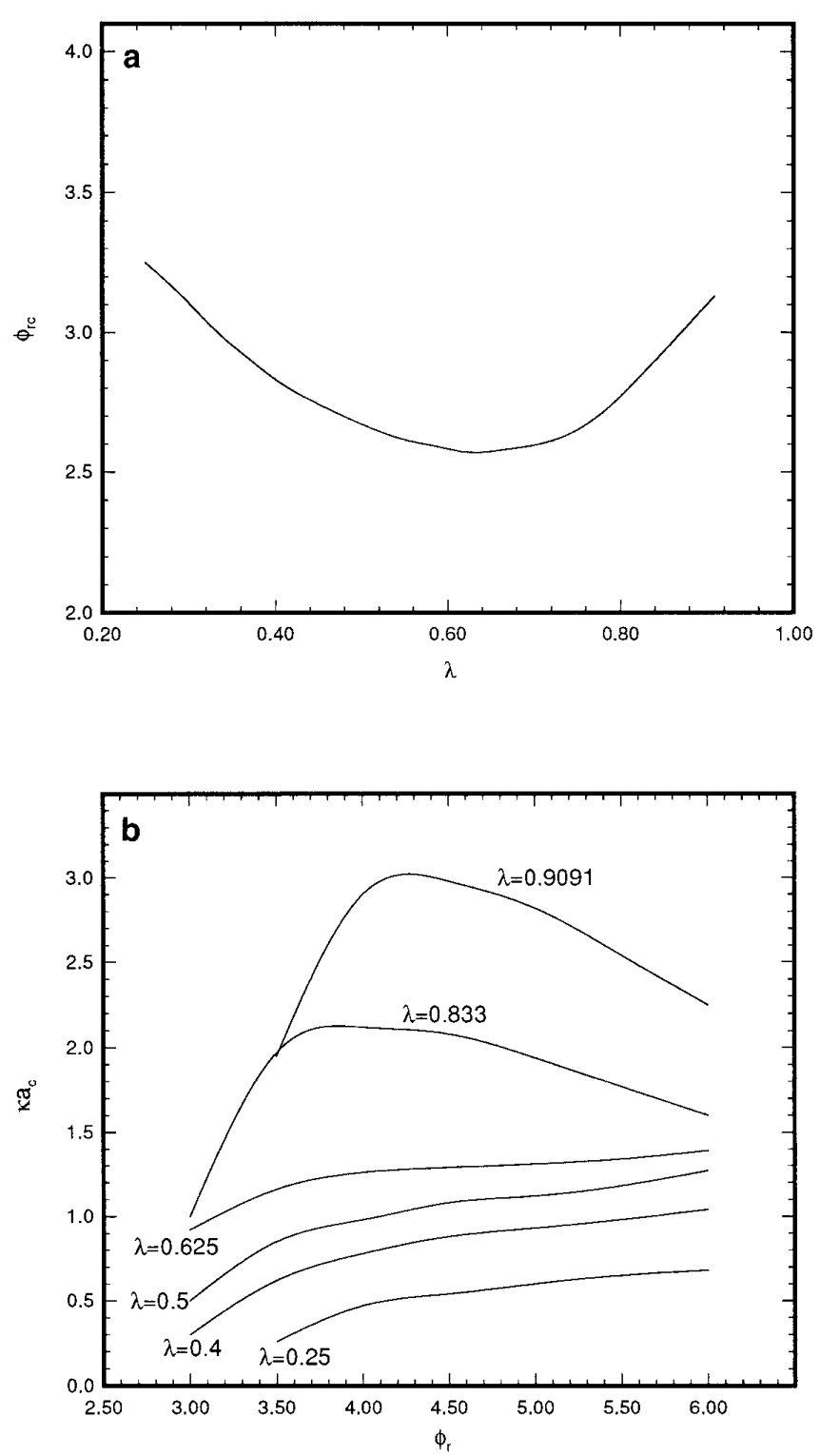

FIG. 5. (a) Variation of $\phi_{r c}$, the scaled surface potential of particle at which the mobility has the minimum, as a function of $\lambda$. (b) Variation of $\kappa a_{c}$, the value of $\kappa a$ at which the mobility has the minimum, as a function of $\phi_{r}$ at various $\lambda$. The particle is positively charged, and the cavity is uncharged. Parameters used are $\mathrm{Pe}_{1}=\mathrm{Pe}_{2}=0.1$ and $\alpha=1$.

influence on the flow field. The strength of the electroosmosic flow is increased, and the mobility of a particle has a maximum. This phenomenon is not observed in the case where the particle is charged and the cavity is uncharged.

The variation of $\phi_{r c}$, the scaled surface potential of cavity at which the mobility has the extreme value, as a function of $\lambda$ is shown in Fig. 8a. This figure reveals that $\phi_{r c}$ increases monotonically with $\lambda$. This is different from the result for the case where the particle is charged and the cavity uncharged. The variation of $\kappa a_{c}$, the value of $\kappa a$ at which the mobility has an extreme value, as a function of $\phi_{r}$ at various $\lambda$ is illustrated in 


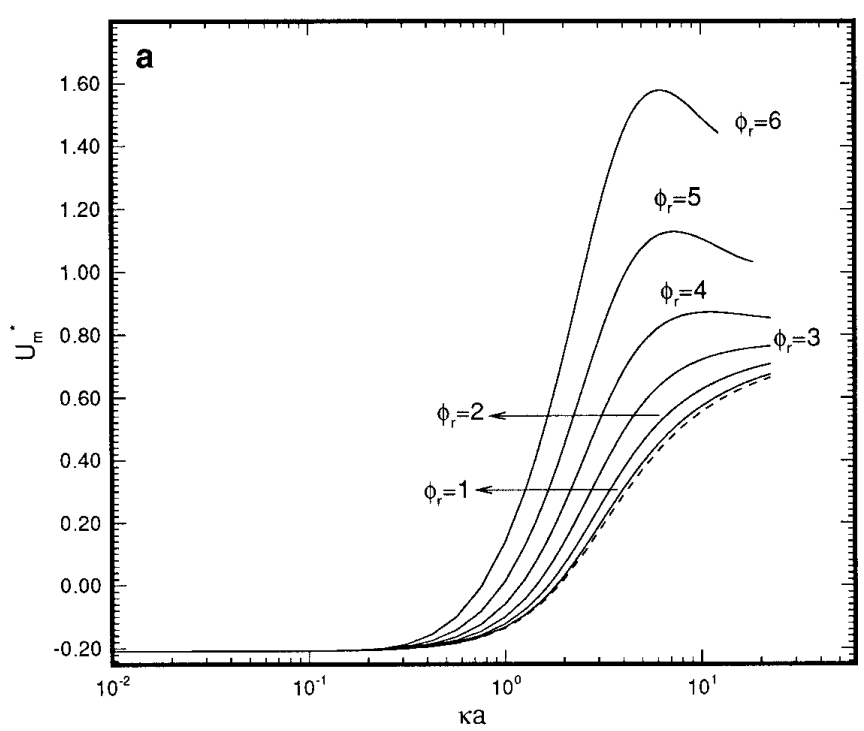

potential of particle, $\phi_{r}$, when both the particle and the cavity are positively charged and when the particle is positively charged and the cavity negatively charged. The results based on the linearized Poisson-Boltzmann equation are also shown in these figures. A comparison between Figs. 2a and $9 \mathrm{a}$ reveals that the $U_{\mathrm{m}}^{*}$ for the case where both the particle and the cavity are positively charged is greater than that for the case where the particle is positively charged and the cavity uncharged. This is because that the electroosmotic flow induced by the cavity is in the same direction as that of

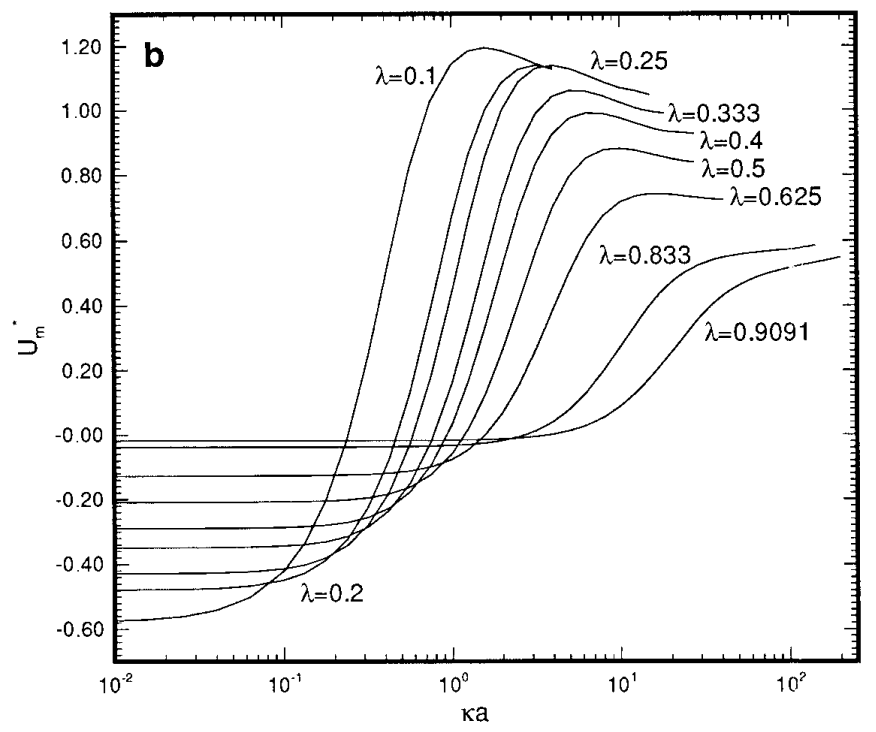

FIG. 6. (a) Variation of the scaled mobility of a particle, $U_{\mathrm{m}}^{*}$, as a function of $\kappa a$ at various scaled surface potential of cavity, $\phi_{r}\left(=\zeta_{b} z_{1} e / k T\right)$, for the case $\lambda=0.5$. The particle is uncharged, and the cavity is positively charged. The dashed line denotes the result based on (14). (b) Variation of $U_{\mathrm{m}}^{*}$ as a function of $\kappa a$ at various $\lambda$. Key: $\mathrm{Pe}_{1}=\mathrm{Pe}_{2}=0.1$ and $\alpha=1$.

Fig. 8b. As can be seen from this figure, $\kappa a_{c}$ decreases monotonically with the increase in $\phi_{r}$.

\subsection{Both Particle and Cavity Are Charged}

If both the particle and the cavity are charged, the electrophoretic behavior of a particle can be deduced from the results of the above two cases. Figure $9 \mathrm{a}$ and $b$, for example, show respectively the variations of the scaled mobility of a particle, $U_{\mathrm{m}}^{*}$, as a function of $\kappa a$ at various scaled surface
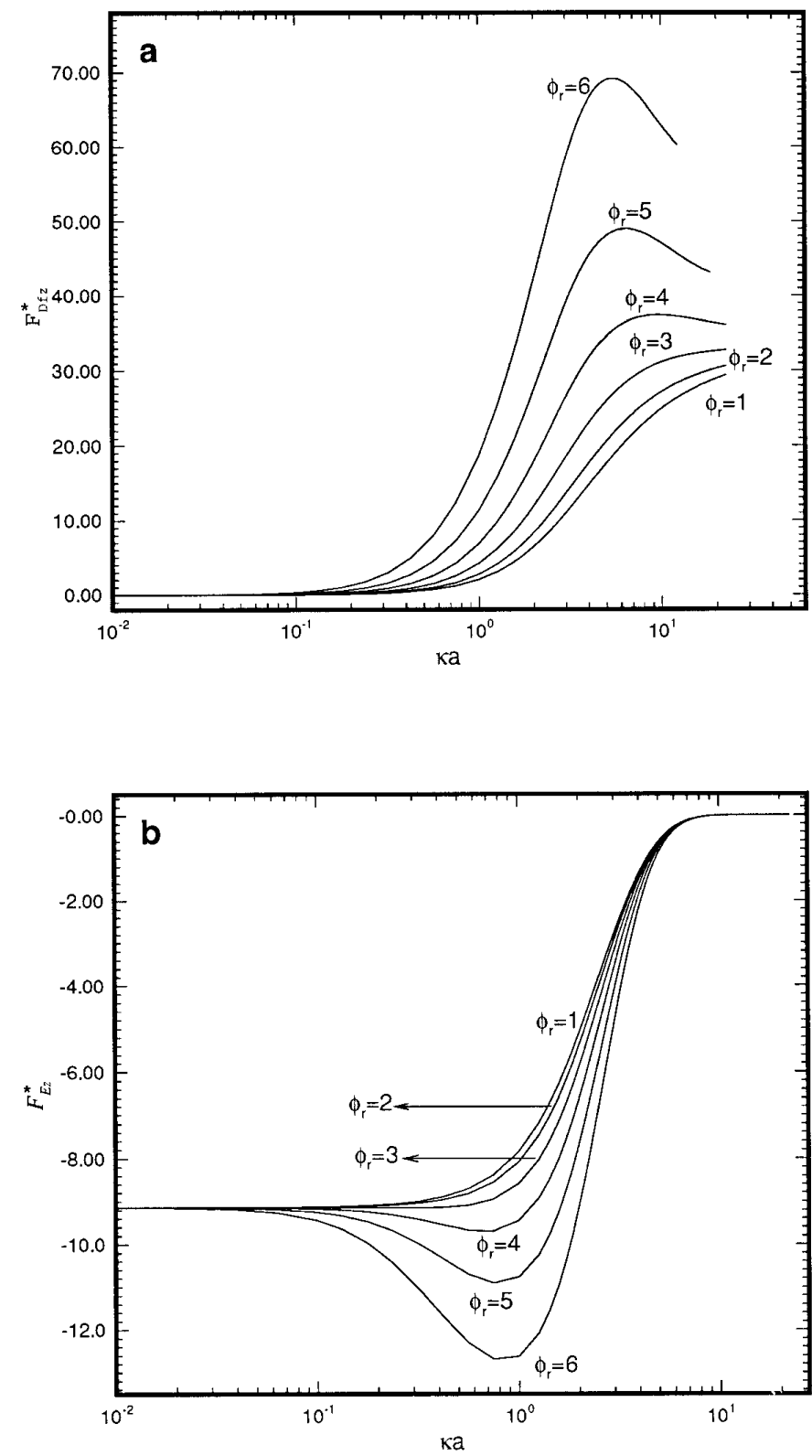

FIG. 7. (a) Variation of $F_{D_{f z}}^{*}$ as a function of $\kappa a$ at various scaled surface potential of cavity, $\phi_{r}$. (b) Variation of $F_{E_{z}}^{*}$ as a function of $\kappa a$ at various $\phi_{r}$. The particle is uncharged, and the cavity is positively charged. Key: same as Fig. 6 except $\lambda=0.5$. 


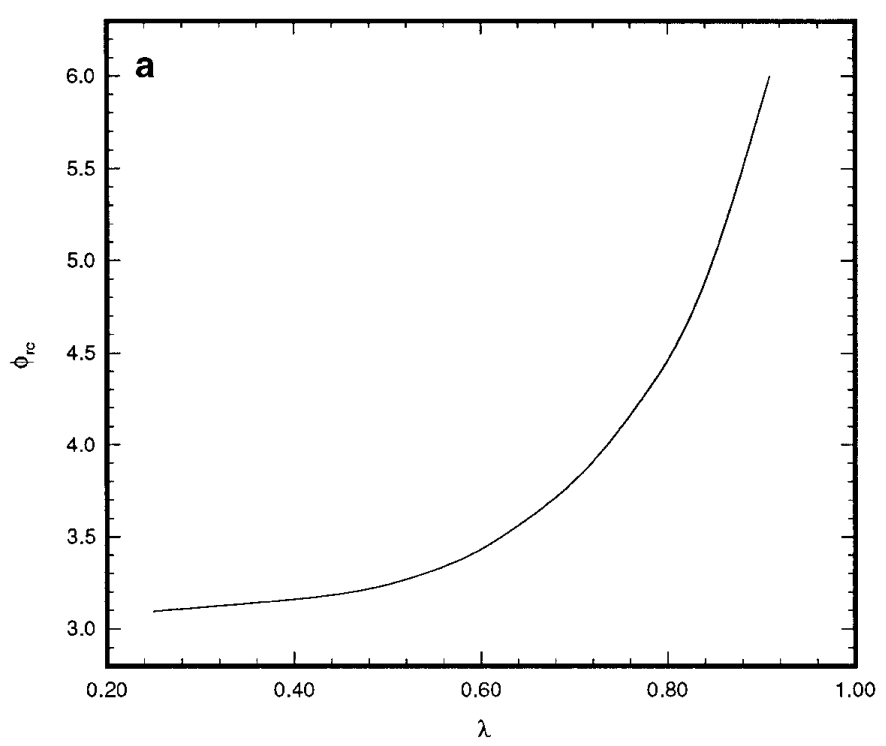

maximum and a minimum for a high $\phi_{r}$. If $\phi_{r}$ is low, the positive charge induced on particle surface due to the presence of the negatively charged cavity is limited. In this case, $U_{\mathrm{m}}^{*}$ decreases first with the increase in $\kappa a$ for a small to medium $\kappa a$, and then increases with a further increase in $\kappa a$ due to the influence of the electroosmotic flow. If $\phi_{r}$ is high, the positive charge induced on particle surface is appreciable, and $U_{\mathrm{m}}^{*}$ increases with the increase in $\kappa a$ for a small to medium $\kappa$. As $\kappa a$ becomes large, the electroosmotic flow,
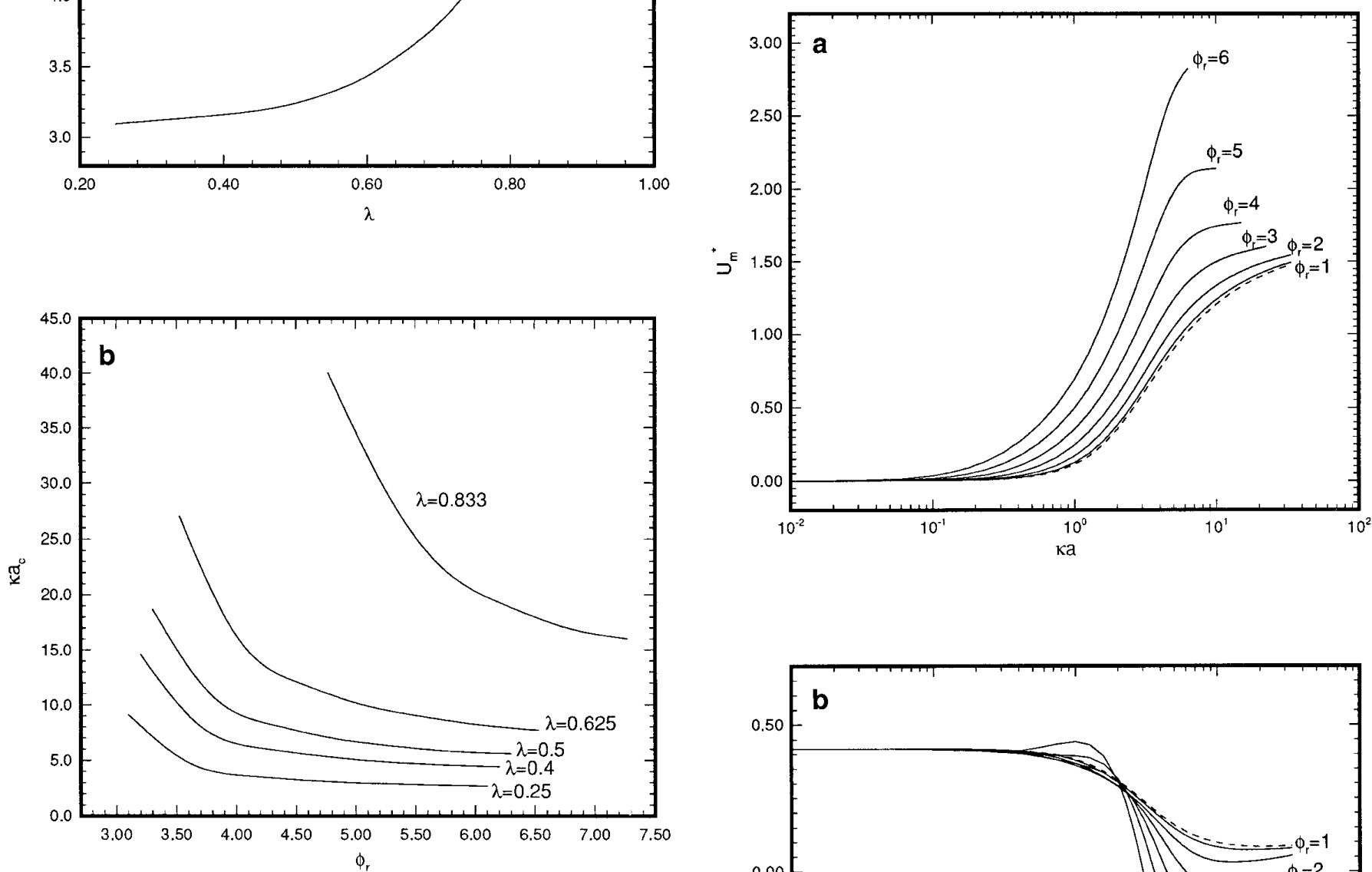

FIG. 8. (a) Variation of $\phi_{r c}$, the scaled surface potential of cavity at which the mobility has the maximum, as a function of $\lambda$. (b) Variation of $\kappa a_{c}$, the value of $\kappa a$ at which the mobility has the maximum, as a function of $\phi_{r c}$ at various $\lambda$. The particle is uncharged, and the cavity positively charged. Key: same as Fig. 6.

the electric force. Also, due to the presence of the electroosmotic flow, the minimum of the variation of $U_{\mathrm{m}}^{*}$ as a function of $\kappa a$ at a high $\phi_{r}$ in Fig. 2a vanishes, as can be seen in Fig. 9a. Similar to the case of Fig. 2a, the higher the $\phi_{r}$, the greater the deviation of the result based on the linearized Poisson-Boltzmann equation from the present result. In contrast to Fig. 2a, however, the deviation is positive. Figure $9 \mathrm{~b}$ suggests that, if the particle is positively charged and the cavity negatively charged, the variation of $U_{\mathrm{m}}^{*}$ as a function of $\kappa a$ has a minimum for a low $\phi_{r}$, and a

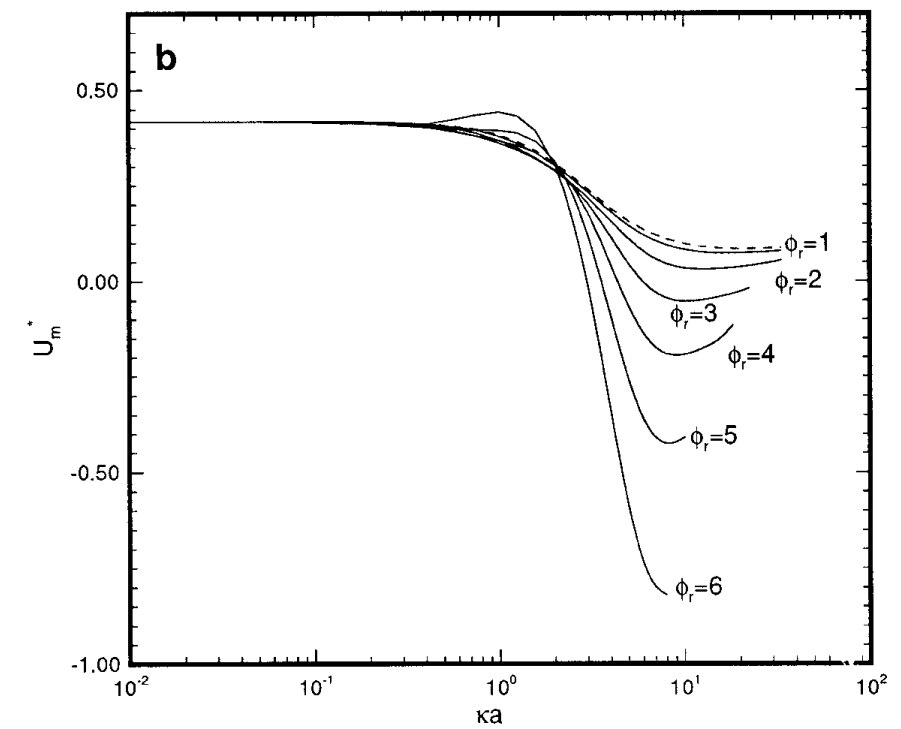

FIG. 9. Variation of the scaled mobility of a particle, $U_{\mathrm{m}}^{*}$, as a function of $\kappa a$ at various scaled surface potential of particle, $\phi_{r}$. (a) Both the particle and the cavity are positively charged, $\phi_{1}^{*}\left(r^{*}=1\right)=\phi_{1}^{*}\left(r^{*}=1 / \lambda\right)=1$; (b) the particle is positively charged and the cavity is negatively charged, $\phi_{1}^{*}\left(r^{*}=1\right)$ $=1, \phi_{1}^{*}\left(r^{*}=1 / \lambda\right)=-1$. The dashed line represents the result based on the linearized Poisson-Boltzmann equation. Key: same as Fig. 2. 
which has an opposite direction to the electric force, becomes dominant, $U_{\mathrm{m}}^{*}$ decreases, and, therefore, it has a maximum.

It should be pointed out that the numerical scheme adopted in the present study is inefficient if $\kappa a$ is greater than about 10 . This is because when $\kappa a$ is large, the double layer near a charged surface is thin, and the spatial variation of the electrical potential inside becomes very steep. In this case, solving Eq. [2] numerically is not recommended, and a semianalytical approach such as perturbation method is suggested.

Although the model system considered here is an imaginary one, it simulates some important cases in practice, such as a particle in a porous medium, and, therefore, it provides valuable information about the effect of a rigid boundary on the electrophoretic behavior of a particle. As pointed out by Zydney (14), however, that since the electroosmotic flow observed in the present system is due to the presence of a closed boundary, the behavior of a particle predicted here may be different from that based on an open system, such as a particle in a cylindrical pore, or a particle near a planar surface.

\section{ACK NOWLEDGMENT}

This work is supported by the National Science Council of the Republic of China.

\section{REFERENCES}

1. Hunter, R. J., in "Zeta Potential in Colloid Science." Academic Press, New York, 1981.

2. Hunter, R. J., in "Foundations of Colloid Science," Vols. I and II. Clarendon Press, Oxford, 1989.
3. Smoluchowski, M., Z. Phys. Chem. 92, 129 (1918).

4. Dukhin, S. S., and Derjaguin, B. V., in "Surface and Colloid Science," Vol. 7. Wiley, New York, 1974.

5. O'Brien, R. W., and Hunter, R. J., Can. J. Chem. 59, 1878 (1981).

6. O’Brien, R. W., J. Colloid Interface Sci. 92, 204 (1983).

7. Hückel, E., Phys. Z. 25, 204 (1924).

8. Wiersema, P. H., Loeb, A. L., and Overbeek, J. Th. G., J. Colloid Interface Sci. 22, 78 (1966).

9. O'Brien, R. W., and White, L. R., J. Chem. Soc., Faraday Trans. II 74, 1607 (1978).

10. Allison, S. A., and Nambi, P., Macromolecules 27, 1413 (1994).

11. Allison, S. A., Macromolecules 29, 7391 (1996).

12. Jorgenson, J. W., Anal. Chem. 58, 743A (1986).

13. Keh, H. J., and Anderson, J. L., J. Fluid Mech. 153, 417 (1985).

14. Zydney, A. L., J. Colloid Interface Sci. 169, 476 (1995).

15. Keh, H. J., and Chiou, J. Y., AIChE J. 42, 1397 (1996).

16. Morrison, F. A., and Stuhel, J. J., J. Colloid Interface Sci. 33, 88 (1970).

17. Keh, H. J., and Chen, S. B., J. Fluid Mech. 194, 377 (1988).

18. Keh, H. J., and Lien, L. C., J. Fluid Mech. 224, 305 (1991).

19. Keh, H. J., Horng, K. D., and Kuo, J., J. Fluid Mech. 231, 211 (1991).

20. Feng, J. J., and Wu, W. I., J. Fluid Mech. 264, 41 (1994).

21. Levine, S., and Neale, G. H., J. Colloid Interface Sci. 47, 520 (1974).

22. Kozak, M. W., and Davis, E. J., J. Colloid Interface Sci. 112, 403 (1986).

23. Kozak, M. W., and Davis, E. J., J. Colloid Interface Sci. 127, 497 (1989).

24. Kozak, M. W., and Davis, E. J., J. Colloid Interface Sci. 129, 166 (1989).

25. Ohshima, H., J. Colloid Interface Sci. 188, 481 (1997).

26. Ennis, J., and Anderson, J. L., J. Colloid Interface Sci. 185, 497 (1997).

27. Lee, E., Chu, J. W., and Hsu, J. P., J. Colloid Interface Sci. 196, 316 (1997).

28. Happel, J., and Brenner, H., in "Low-Reynolds Number Hydrodynamics." Martinus Nijhoff, Boston, MA, 1983.

29. Canuto, C., Hussaini, M. Y., Quarteroni, A., and Zang, T. A., in "Spectral Methods in Fluid Dynamics.” Springer-Verlag, New York, 1986. 